\title{
Effects of Exercise Training on the Paracrine Function of Circulating Angiogenic Cells
}

\author{
Authors \\ William S. Evans ${ }^{1}$, Ryan M. Sapp ${ }^{1}$, Katherine I. Kim¹, James M. Heilman'1, James Hagberg1, Steven J. Prior ${ }^{1,2}$
}

\author{
Affiliations \\ 1 Department of Kinesiology, University of Maryland \\ School of Public Health, College Park \\ 2 Baltimore Veterans Affairs Geriatric Research, Education \\ and Clinical Center, Department of Veterans Affairs, \\ Baltimore
}

Key words

microRNA, angiogenesis, extracellular vesicle, cytokine

accepted 15.09.2020

published online $\quad 29.10 .2020$

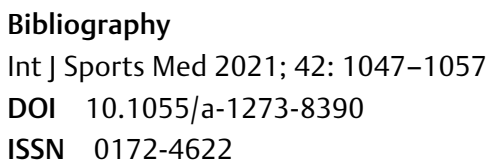

\begin{abstract}
Exercise training has various benefits on cardiovascular health, and circulating angiogenic cells have been proposed as executing these changes. Work from the late 1990s supported an important role of these circulating post-natal cells in contributing to the maintenance and repair of the endothelium and vasculature. It was later found that circulating angiogenic cells were a heterogenous population of cells and primarily functioned in a paracrine manner by adhering to damaged endothelium and releasing growth factors. Many studies have discovered novel circulating angiogenic cell secreted proteins, microRNA and extracellular vesicles that mediate their angiogenic potential, and some studies have shown that both acute and chronic aerobic exercise training have distinct benefits. This review highlights work establishing an essential role of secreted factors from circulating angiogenic cells and summarizes studies regarding the effects of exercise training on these factors. Finally, we highlight the various gaps in the literature in hopes of guiding future work.
\end{abstract}

\section{Introduction}

After the discovery of endothelial progenitor cells (EPCs) in 1997 [1], substantial optimism surrounded these cells for their capacity to engraft into the endothelium and regenerate damaged tissue. However, more recent evidence using progenitor cell transplantation indicated that most of these cells act through paracrine pathways, and also expanded the population to include similar angiogenic bone marrow-derived myeloid precursors, termed circulating angiogenic cells (CAC) [2,3]. These CAC populations are characterized by their ability to adhere to vascular endothelium and secrete various factors that regulate endothelial angiogenesis. Reductions in CAC number and inability to promote angiogenesis have been observed in individuals with various diseases [4] and have been proposed as novel risk factors for cardiovascular diseases (CVD) [5]. Acute exercise increases CAC number and/or function [6] and these exercise-induced changes have been proposed as potential mediators for reducing CVD risk and increasing capillarization in skeletal muscle. A growing body of research supports a contribution of acute exercise in transiently increasing CAC recruitment and paracrine function; however, the chronic effects of exercise training on CAC paracrine function have not been as thoroughly investigated or discussed. This review is intended to 1) summarize current literature supporting a role of CAC paracrine function with a focus on secreted proteins, extracellular vesicles (EVs) and microRNA ( $\mathrm{miR}), 2$ ) review how these factors are altered after 
chronic exercise training, and 3) identify knowledge gaps to guide future investigations of the effects of exercise training.

\section{Defining CACs}

Before discussing the function of CACs, it should be noted that the definition of CACs has expanded to include various cell types such as stem and progenitor cells, monocytes/macrophages [7, 8], T-cells [9], and mesenchymal stem cells [10] ( Table 1). These cells are identified by a broad range of cell surface markers, and entire reviews have been dedicated to the proper quantification, phenotyping and reporting of these cells; thus, there is growing consensus that a comprehensive set of cell surface markers should be used to increase data transparency and reduce ambiguity [24]. In this review, we will use the term CAC to refer to the broad heterogeneous population of primarily myeloid-derived cells capable of inducing angiogenesis, while specific cell surface markers will be identified when referencing specific studies. The most frequently studied subsets of CACs include those expressing CD31 and CD34. CD34 was first identified on hematopoietic stem and progenitor cells and has been linked to regeneration of damaged tissue and de novo production of various cell types. Meanwhile, CD31 is a member of the immunoglobulin superfamily with key roles in executing interactions between circulating cells and endothelial cells [25]. To date, most of our knowledge of the effects of exercise training on the paracrine function of CACs is derived from work on CD34+ and CD34-/CD31 + cells. We now understand that each of these subsets of CACs is comprised of heterogeneous groups of cells, each of which may have a distinct role in vascular maintenance and all of which require future investigation.

\section{CAC Paracrine Function}

EPCs and CACs were initially thought to represent a post-natal cell capable of regenerating vascular tissue and promoting de novo production of blood vessels; however, studies utilizing bone marrow transplantation refuted this, as labeled bone marrow-derived CACs could not be detected within the existing vessels of the recipient [26]. Despite their absence within the vessels, CAC treatment did improve endothelial repair and angiogenesis; furthermore, CACs were observed adjacent to the endothelial repair. These findings suggested that CACs were capable of promoting angiogenesis, albeit in an alternative manner. Subsequent studies hypothesized and later supported the ability of CACs to elicit angiogenesis through the paracrine secretion of pro-angiogenic factors. Several studies have supported this hypothesis, as the effects of treatments involving factors secreted by CACs have been found to elicit similar outcomes as treatment with CACs themselves. While it remains possible that certain CACs may contribute to vascular development through differentiation and engraftment, the majority of data supports CAC paracrine function as the primary determinant of CAC angiogenic potential [27-30]. The following sections describe the key CAC paracrine factors identified to date.

\section{CAC-derived proteins}

The angiogenic potential of CACs is partially determined by the relative amount and type of protein secreted by CACs. Many of the proteins released by CACs have been identified and include isoforms of vascular endothelial growth factor (VEGF), basic fibroblast growth factor (bFGF), angiopoietin-1 (Ang-1) [31], hepatocyte growth factor (HGF), granulocyte colony stimulating factor

- Table 1 Definitions and Terminology Associated with Circulating Angiogenic Cells.

\begin{tabular}{|c|c|c|c|}
\hline Cell type & Surface marker & Reference & Definition \\
\hline $\begin{array}{l}\text { Circulating angiogenic } \\
\text { cell (CAC) }\end{array}$ & Varies & $\begin{array}{l}\text { Fadini et al. [11] } \\
\text { Hill et al. [12] }\end{array}$ & $\begin{array}{l}\text { Also used as a broad term often to define a variety of isolated } \\
\text { circulating cells with angiogenic potential. } \\
\text { Also used in some literature to define colony forming units } \\
\text { (CFUs), a heterogeneous population of cells derived from cell } \\
\text { culture of peripheral blood mononuclear cells and re-plating of } \\
\text { non-adherent cells to form colonies. }\end{array}$ \\
\hline $\begin{array}{l}\text { Mononuclear cells } \\
\text { (MNCs) }\end{array}$ & & & Heterogeneous mixture of bone marrow-derived cells. \\
\hline EPC & $\begin{array}{l}\mathrm{CD} 34+/ K D R+ \\
\mathrm{CD} 34+/ K D R+/ C D 133+ \\
\mathrm{CD} 34+/ K D R+/ C D 45- \\
\mathrm{CD} 133+/ K D R+\end{array}$ & $\begin{array}{l}\text { Asahara et al. [13] } \\
\text { Fadini et al. [11] }\end{array}$ & $\begin{array}{l}\text { Cells expressing surface markers for both immature progenitor } \\
\text { cells }(\mathrm{CD} 34+\text { or } \mathrm{CD} 133+) \text { and endothelial cells }(\mathrm{KDR}+) \text { usually } \\
\text { identified using flow cytometry on freshly isolated circulating } \\
\text { MNCs. }\end{array}$ \\
\hline $\begin{array}{l}\text { Circulating progenitor } \\
\text { cell / Hematopoietic } \\
\text { progenitor cells }\end{array}$ & $\begin{array}{l}\text { CD34+ } \\
\text { CD133+ }\end{array}$ & $\begin{array}{l}\text { Rigato et al. } \\
\text { Fadini et al. [11] } \\
\text { Bielak et al. [14] }\end{array}$ & $\begin{array}{l}\text { Immature bone-marrow-derived cells, mostly of hematopoietic } \\
\text { origin associated with cardiovascular health. Includes EPCs and } \\
\text { other progenitor cells found in circulation. }\end{array}$ \\
\hline Circulating CD31+ cell & $\mathrm{CD} 31+$ & $\begin{array}{l}\text { Kim et al. [15] } \\
\text { Kim et al. [16] } \\
\text { Erdgruegger et al. [17] } \\
\text { Goon et al. [18] }\end{array}$ & $\begin{array}{l}\text { Circulating CD31-expressing hematopoietic and endothelial cells } \\
\text { present in peripheral blood with vasculogenic and angiogenic } \\
\text { properties. Includes mature endothelial cells in circulation. }\end{array}$ \\
\hline Angiogenic T cells & $\begin{array}{l}\mathrm{CD} 31+/ \mathrm{CD} 3+ \\
\mathrm{CD} 31+/ \mathrm{CD} 3+/ \mathrm{CXCR} 4+\end{array}$ & $\begin{array}{l}\text { Kushner et al. [19] } \\
\text { Hur et al. [20] }\end{array}$ & Subpopulation of T-cells with vasculogenic properties. \\
\hline Angiogenic monocytes & $\begin{array}{l}\mathrm{CD} 31+/ \mathrm{F} 4 / 80 \\
\mathrm{CD} 31+/ \mathrm{CD} 14+\end{array}$ & Kim et al. [21] & \\
\hline Other & CD62E + & Waller et al. [22] & $\begin{array}{l}\text { Non-progenitor peripheral blood mononuclear cells with } \\
\text { angiogenic properties. }\end{array}$ \\
\hline
\end{tabular}


(G-CSF), stromal cell-derived factor-1 (SDF-1), interleukin-8 (IL-8), and transforming growth factor- $\beta 2$ (TGF- $\beta 2$ ) [7, 32]. Current evidence suggests complex interplay among these factors that ultimately governs angiogenesis.

Initially, EPCs were described as targets of secreted factors, namely VEGF. Indeed, activation by VEGF promotes CAC proliferation and nitric oxide (NO) release [13, 33]. However, investigators illustrated a paracrine role of EPCs as hypoxic pre-conditioning increased VEGF production and improved blood flow restoration after EPC transplantation during hindlimb ischemia [34]. Furthermore, VEGF was identified as more highly expressed by EPC and CD14 + cells compared with human umbilical vein endothelial cells (HUVEC) [32]. Moreover, treatment of EPC conditioned media (CM) with an anti-VEGF antibody reduced the CM's ability to induce endothelial cell migration. This early work established a clear role of VEGF, but later found that VEGF operated in tandem with other factors. For example, the proangiogenic paracrine effects of EPC on retinal endothelial cells are reduced after blockade of IL-8, but not of VEGF [35]. This study replicated these findings in vivo as vascular repair seemingly relied on the action of IL- 8 to increase endothelial permeability by phosphorylating extracellular signal-regulated kinase (ERK) through the activation of the C-X-C motif chemokine receptor (CXCR) -2 and VEGF receptor 2 (VEGFR2) [36]. These results also suggest potential overlapping paracrine functions of proteins, considering IL- 8 is able to act through VEGFR2 and compensate for the absence of VEGF.

EPCs secrete relatively high levels of SDF-1, and investigators have revealed a paracrine role for SDF-1 via its receptor CXCR4 on endothelial cells $[37,38]$. Exposure to SDF-1 enhances a range of angiogenic processes and results in augmented repair of mouse hindlimb ischemia [37] that can be prevented with highly specific antagonists to CXCR4 [37, 39]. Data suggest that SDF-1 works in tandem with VEGF to mediate CAC paracrine effects as inhibition of both, but not each individual protein, caused significant reductions in repair of hindlimb ischemia [38]. This may reflect redundancies in the signaling pathways of these two cytokines and/or the ability of VEGF to upregulate SDF-1 expression [40] and vice-versa.

The aforementioned cytokines represent only a few of the known soluble proteins involved in the paracrine action of CACs. A more complete picture has been constructed by multiplex arrays and proteomics analyses [41-43]. These studies have identified as many as 647 [43] or as few as 82 proteins [42] in the secretome of CACs. In a recent study by Felice et al. [43], 83 proteins were differentially secreted in response to hypoxia, of which a number of angiogenic proteins were upregulated and anti-angiogenic proteins were downregulated, indicating the putative role of CAC paracrine function in vascular homeostasis. It was also found that $\mathrm{CM}$ collected from EPCs cultured under hypoxic conditions had protective, anti-apoptotic effects on HUVECs, even after neutralization of four of the most highly expressed cytokines (VEGF, HGF, matrix metallopeptidase-9, IL-8) [28]. Collectively, these data support the notion that a number of proteins are likely involved in the paracrine angiogenic function of CACs.

While the paracrine action of CACs is rapidly becoming well established, it is important to note the plasticity and alteration of these actions in health and disease. For example, krueppel-like factor 10 (an effector of CXCR4 expression and paracrine signaling) expression is reduced by $\sim 70 \%$ in CD34 + /VEGFR2 + cells of peripheral arterial disease (PAD) patients, probably contributing to some of the reduced paracrine functions often observed in this and similar unhealthy/diseased populations [44]. Cultured CD34 + /VEGFR2 + cells from patients with permanent atrial fibrillation, a group with high rates of endothelial dysfunction, secreted lower levels of VEGF and NO than age- and sex-matched controls [45]. CM from CAC of critical limb ischemia patients could not elicit endothelial migration to the same degree as CAC-CM of healthy, age-matched controls [46]. EPCs expanded from mononuclear cells of obese individuals displayed lower levels of secreted IL-8 and monocyte chemoattractant protein-1 (MCP-1) than lean individuals [47]. In diabetic individuals, CD34 + cells were found to secrete substantially lower levels of stem cell factor, HGF, and thrombopoietin, along with elevated levels of MCP-1, granulocyte monocyte-colonystimulating factor, IL-6, and tumor necrosis factor-alpha (TNF- $\alpha$ ) as compared to non-diabetic subjects [48]. The secreted factors from CD34 + CACs from patients with diabetes were unable to stimulate angiogenesis or regulate vascular reactivity [48]. These findings suggest that CAC paracrine function is sensitive to health status and the extracellular environment, providing a putative target for therapeutic pharmaceutical or lifestyle interventions such as exercise training.

Indeed, preconditioning CACs in hypoxia has become a common technique to increase their proangiogenic functions/secretions before subsequent use in therapies or experiments, although not all studies have reported beneficial effects [38]. Interestingly, in addition to increasing the secretion of VEGF, SDF-1, chemokine ligand 1 , and macrophage migration inhibitory factor (MIF), hypoxia upregulated the expression of CXCR2 and CXCR4 on the surface of EPCs, potentiating a beneficial autocrine loop [49]. Other proteins and pathways regulating CAC paracrine function have been identified, some of which overlap with pathways established as contributing to the overall benefits of exercise training. These include AMP-activated protein kinase [50], peroxisome proliferator-activated receptor $-\delta[51]$, signal transducer and activator of transcription [52], krueppel-like factor 10 [53], and p38 mitogen-activated protein kinase (MAPK) [47]. As one example, AICAR (an AMP analog which stimulates AMP-activated protein kinase) stimulation of EPCs induced greater release of the pro-angiogenic VEGF, IL-8, and SDF-1 into media and enhanced HUVEC migration in a gap closure assay [50].

Lastly, there is evidence to suggest that CD34 + CACs work synergistically with other cell types including endothelial cells, monocytes (CD14 + cells), and mesenchymal stromal cells (MSCs) to amplify paracrine effects. Co-culturing CD34 + cells with HUVECs led to markedly higher media concentrations of IL-8, IL-6, macrophage inhibitory protein (MIP)- $1 \alpha$, MIP-1 $\beta$, and MCP-1 compared to culturing either cell type alone [54]. In addition, the complementary effects of paracrine factors from MSCs and EPCs were later revealed as the combination of CM from both cell types greatly enhanced proliferation of endothelial cells compared to CM of either cell type alone [55]. Interestingly, CD14 + monocytes previously incubated in CD34 + CM had enhanced production of MCP-1, FGF-2, HGF and VEGFa, showing that CACs can stimulate paracrine functions of other circulating cells [56]. In vivo, the co-implantation of both CD34 + and CD14 + cells into cell-loaded matrigel plugs led to 
greater microvessel formation than either individual cell type [56]. Incorporation of either cell type into the vasculature was not observed, implying the mediation of paracrine factors. Indeed, when the group of growth factors IL-8, MCP-1, FGF-2, HGF and VEGFa were neutralized, the stimulatory effects on neovascularization were abolished [56].

In summary, the literature supports the role of CAC-derived proteins in contributing to angiogenesis and maintenance of the endothelium. Much of this angiogenic potential is attributable to traditional growth factors such as VEGF, FGF, HGF, bFGF and G-CSF; however, many of these cytokines and their targets also are affected by inflammatory factors such as IL-6, IL-8, signal transducer and activator of transcription-3, MCP-1, and MIF-1. Thus, there is likely complex interplay among these cytokines that will require further study. Moreover, these proteins do not comprise the entirety of CAC paracrine factors as recent evidence implicates a discrete role of other factors such as miRs and EVs.

\section{CAC-derived microRNAs}

In addition to proteins, CACs release microRNAs (miRs) to enact their paracrine effects. MiRs are a class of short, noncoding RNAs that post-transcriptionally regulate the expression of target genes. By binding to complementary sequence(s) in the 3' untranslated region of target mRNAs, the mature miR acts as a guide for an associated miR-induced silencing complex that interferes with translation [57-59]. Because perfect sequence alignment is not necessary for miRs to exert an effect, each miR is able to directly target several mRNAs. In addition, multiple miRs may share the same mRNA target, thus contributing to the complexity of miR effects on paracrine function [57].

MiR-126 is the most abundant miR within endothelial cells, where its roles in angiogenesis and vascular repair have been well characterized [60]. The pro-angiogenic miR-126 has more recently emerged as a critical regulator of CAC function and may, in part, regulate $C A C$ paracrine function by targeting inhibitors of the VEGF signaling pathway [61]. Investigators have identified SDF-1 as a direct target of miR-126 in EPCs and determined that miR-126 improves EPC migration through the SDF-1/CXCR7 pathway [62]. MiR126 was also confirmed to target PIK3R2 in EPCs and consequently to activate the PI3K/Akt pathway and inhibit the FOXO3/SMAD4 signaling pathways in TGF 31 -stimulated EPCs [63]. The overexpression of miR-126 in EPCs resulted in increased proliferation, migration and angiogenic functions through activation of the PI3K/Akt/ eNOS pathway, in addition to beneficial alteration of NO and ROS levels [64]. The effects of miR-126 overexpression appear to persist in vivo, as transplantation of miR-126 transfected EPCs increased angiogenesis and cerebral blood flow in a mouse model of ischemic stroke [62, 64].

Additional evidence suggests that EPC production or secretion of paracrine factors may be mediated by the miR-126 response to the extracellular environment. Culturing EPCs in media supplemented with high glucose or advanced glycation end products (AGEs) decreased their expression of miR-126 and increased ROS production and secretion of inflammatory cytokines IL- 6 and TNF- $\alpha$; however, miR-126 overexpression was able to attenuate these effects [65]. Furthermore, Jakob et al. [66] observed lower miR-126 expression coinciding with higher mRNA and protein expression of its direct target sprouty-related, EVH domain-containing protein-1 in circulating CD34 + cells and cultured endothelial outgrowth cells (EOC) of CHF patients compared to those of healthy controls. Injection of the EOCs into mice following acute MI revealed inferior ability of CHF patients' cells to improve cardiac function and induce neovascularization; however, transfection of the CHF patient-derived EOCs with a miR-126 mimetic significantly improved these functions [66].

In regard to other miRs, the CD34 + cells of CHF patients also contained lower miR-130 and higher levels of its target homeobox protein 5, which may reduce angiogenic functions by downregulating VEGFR2, Ephrin A1, hypoxia-inducible factor 1-alpha (HIF1 $\alpha$ ), and cyclooxygenase- 2 , and by upregulating antiangiogenic genes including thrombospondin-2 [66]. An additional target of miR-130 is runt-related transcription factor 3 (Runx3), which negatively regulates EPC functions by suppressing VEGF expression [67]. Upregulation of miR-130 decreased Runx3 expression and positively regulated ERK/VEGF and Akt in EPCs [67]. The EPCs of people with type 2 diabetes also exhibit low levels of miR-130 and elevated Runx3, which contributes to their dysfunction [67].

MiR-21 also is expressed in CACs and its expression is altered in the presence of a variety of factors including age, CVD, asymmetrical dimethylarginine (ADMA), shear stress, and hypoxia [65-67]. MiR-21 may have overlapping function with miR-126 in mature endothelial cells due to its ability to downregulate the expression of phosphatase and tensin homolog, an antagonist of the PI3K/Akt/ eNOS pathway [68-70]; however, the same may not be true when assessing the effects of miR-21 on CACs. For example, the NOS inhibitor ADMA, which is elevated in coronary artery disease (CAD) patients, was shown to induce CAC dysfunction in a miR-21 dependent manner [71]. Specifically, ADMA caused a three-fold upregulation of miR-21, which reduced EPC migration by increasing ROS production and decreasing NO bioavailability [70]. When CACs of CAD patients were transfected with anti-miR-21, migratory capacity was restored, justifying miR-21 as a clinically relevant target with therapeutic potential [70]. Additionally, miR-21 has been found to both suppress EPC proliferation by targeting WWP1, a negative regulator of TGF $\beta$ signaling, and promote senescence by targeting $\mathrm{Hmga} 2$ in conjunction with miR-10a * [69, 102].

While the aforementioned miRs have received the most attention in the literature, a number of other miRs may play roles in the paracrine function of CACs. For example, miR-142 acts to increase VEGF expression by downregulating another of its inhibitors, a disintegrin and metalloproteinase with thrombospondin motifs - 1 , resulting in greater eNOS and NO production [72]. Conversely, miRs-15a and 16 both directly target VEGF, and the CACs of critical limb ischemia patients displayed greater expression of these two miRs which were responsible for their reduced angiogenic function [73]. Evidence suggests that antagonism of miR-16 improves the angiogenic functions of CACs, potentially through increased secretion of proangiogenic factors such as IL-8 [74]. Another direct antagonist of VEGF and negative regulator of angiogenesis is miR-361, which is upregulated in EPCs and plasma of CAD patients [75]. Likewise, miR-138 directly targets HIF-1 $\alpha$ and is thereby negatively regulated in response to senesence in endothelial cells. Similarly, in both CACs and plasma from CHF patients, miR146a expression was 1000 -fold and 2 -fold greater, respectively, 
compared with healthy controls [76]. Together, these studies show that several miRs that modulate paracrine function of CACs are aberrantly expressed in CACs from individuals with various cardiometabolic diseases. Still, research has been performed on a limited number of miRs, and the roles of many others remain to be established.

\section{CAC-derived extracellular vesicles and cargo}

EVs also appear to play a substantial role in CAC paracrine function, as proteins and miRs are often packaged within EVs for transport. EVs are formed by budding or blebbing from the plasma membrane. These vesicles act as intercellular communicators released from CAC and other cell types and contain a wide range of cargo, such as proteins, mRNAs, microRNAs (miRs), and DNA [77, 78]. Variability exists in EV nomenclature, but they are generally denoted by their size (50-150 nm) and expression of specific markers (tetraspanins: CD9, CD81, flotilin 1 and 2), but other cargo carriers such as microvesicles, apoptotic bodies, migrasomes, and arrestin-domain-containing protein-1 mediated microvesicles are released from cells and have overlapping characteristics [79]. Despite their heterogeneity and the associated challenges in characterizing them, CAC-derived EVs (CAC-EVs) have shown promise as mediators of the paracrine function of CACs $[80,81]$.

The content and subsequent function of CAC-EVs are regulated by both their source and microenvironment. One of the first studies using CAC-EVs generated in vitro from CD133 + /VEGFR2 + I CD34 + /CD45-dim/null CACs clearly illustrated their angiogenic potential; these EVs were capable of horizontal mRNA transfer into endothelial cells to reprogram them for angiogenesis [80]. Furthermore, CD34 + cell EVs were shown to have angiogenic function similar to CD34 + cells alone and superior benefits on endothelial viability and proliferation [82]. These studies implicated a specific role of $\mathrm{CD} 34+$ exosomes in executing the angiogenic function of CD34 + cells, and it was hypothesized that the cargo responsible for these changes were pro-angiogenic miRs-126 and -130a, which were highly concentrated within the CAC-EVs [82].

CAC-EVs contain a plethora of cargo including proteins and mRNA, but early work implies that miR-126 plays a distinct role in mediating CAC-EV function [82]. In response, studies utilizing in vivo models of angiogenesis have started to address this gap. For example, treatment with CAC-EVs was performed in an animalmodel of peripheral ischemia and improved motor function, perfusion, limb salvage, and capillary density [81]. Upon assessment of the CAC-EVs, protein content was not substantially different; however, CAC-EVs had distinctly different miR profiles, implicating miRs as the primary determinant of the proangiogenic potential of CAC-EVs. Further investigation supported earlier findings as miR126 knock-down abrogated the angiogenic capacity of CAC-EVs in vivo and in vitro. Finally, this study supported a direct transfer of miR- 126 by illustrating increased miR- 126 within target endothelial cells and specific changes in expression of its target mRNA. Overall, these findings provided evidence for an explicit role of EVencapsulated miRs, particularly miR-126, in mediating the proangiogenic and potential therapeutic effects of CAC-EVs in vivo.

Though CAC-EVs have been investigated for their angiogenic function, it is apparent that the CAC-EVs of individuals with chronic diseases can be a source and/or target of dysfunction. Some stud- ies have shown that damaged endothelial cells release apoptotic bodies that subsequently communicate with CACs to induce dysfunction $[83,84]$. Though this finding requires further support, it strongly suggests that CAC-EVs are dysregulated in chronic diseases. For example, decreased miR-126 expression within CAC-EVs has been identified as a significant indicator of disease severity in those with chronic diseases. This is exemplified by findings in patients with type 2 diabetes who have reduced miR-126 expression within CACs. This reduced expression is mirrored in CAC-EVs, as patients with uncontrolled diabetes carried less miR-126 in their CAC-EVs than healthy controls and even those with well-controlled diabetes [85]. Furthermore, in participants with severe diabetes, CAC-derived EVs induced ROS production and apoptosis and reduced $C A C$ migration; however, restoring miR-126 content within CAC-EVs was found to improve the function of CACs. In addition, CAC-EVs enriched with pro-inflammatory and anti-angiogenic proteins along with miR-375 (an inhibitor of the PI3k/Akt pathway) exhibited impaired angiogenic capacity when compared to wild-type controls [86]. These findings support the likelihood that the content of CAC-EVs exist on a spectrum from unhealthy (antiangiogenic) to healthy (proangiogenic) and fuel the rationale for interventions such as exercise training to alter their content and function.

\section{Effects of Exercise Training on CAC Paracrine Functions}

\section{CAC-derived proteins}

Though many studies have identified the contributions of specific proteins to the paracrine functions of CACs, there are considerably less data describing how the $C A C$ release of soluble proteins and their subsequent paracrine effects are modified in response to exercise training. To date, much of our understanding with regard to the paracrine function of CACs after exercise training comes from studies on CD34 + and CD34-/CD31 + CACs previously performed by our group $[87,88]$. In one of the first studies, CACs were isolated from young, healthy participants who were either inactive or engaged in regular endurance exercise training [88]. The CM harvested from the CACs of inactive individuals from both CAC subtypes resulted in shorter and less complex endothelial tube formation in vitro, suggesting functional impairments of the paracrine angiogenic capacity of these CACs ( $>$ Fig. 1). Through proteomic analysis, S100A8 and S100A9 were identified as proteins potentially responsible for this impairment, and differences in the concentration of these proteins were verified in the CM of CD34-/CD31 + cells, but not in the CM of CD34 + cells. Given previous work by our group, we hypothesized that the differences in tube formation would arise as a function of intracellular redox balance. However, this was not found to be the case in the young healthy participants enrolled in this study. To assess whether the cross-sectional nature of the study or the younger age of the study population played a role in the lack of observed differences in intracellular redox balance, our group recruited older sedentary adults for a short-term, vigorous aerobic exercise intervention ( 10 days of daily training at $70 \%$ VO2max for $60 \mathrm{~min}$ ) [89]. Though the numbers of CD34 + $\mathrm{KDR}+$ and KDR + CAC subtypes and endothelial function increased 


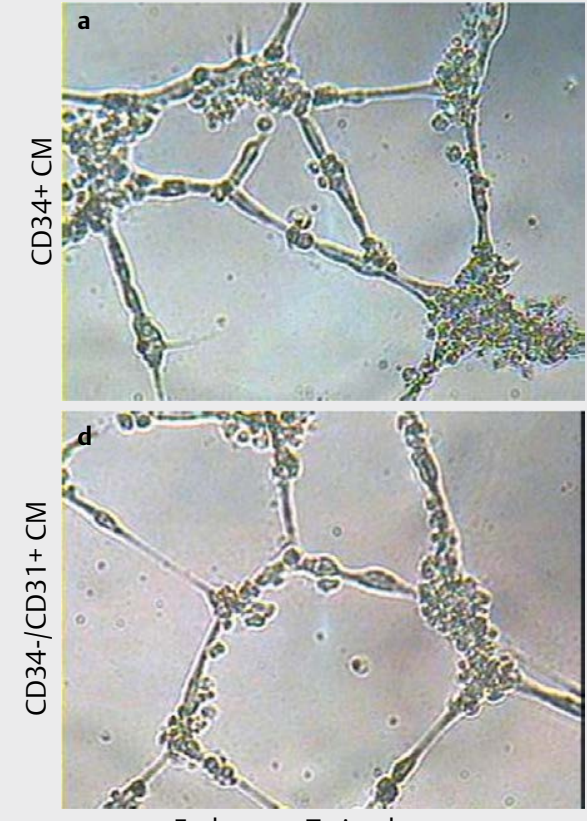

Endurance Trained
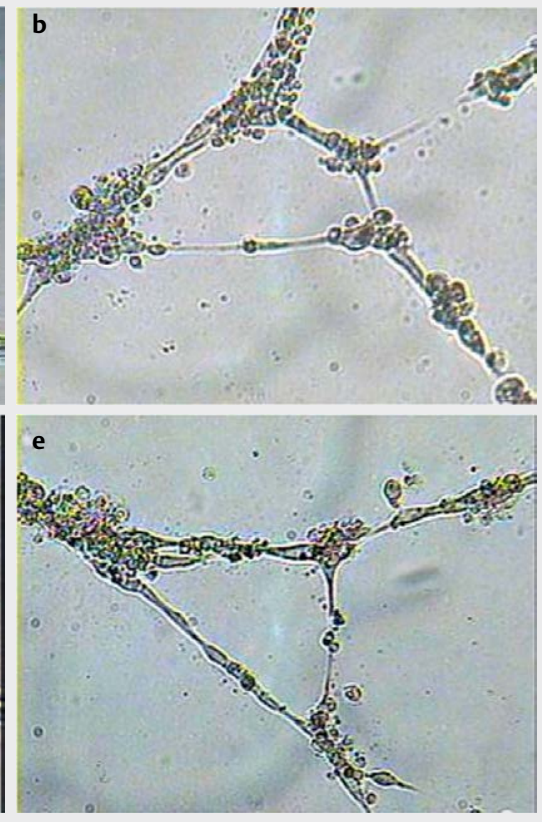

Active

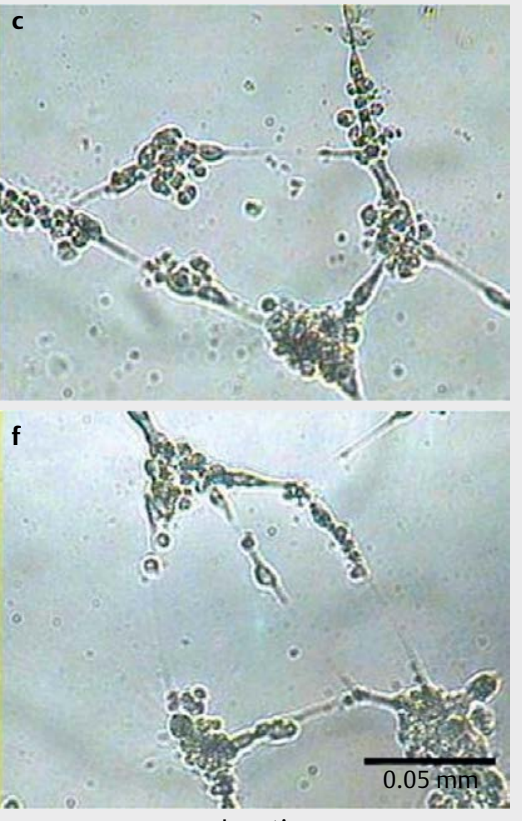

Inactive

-Fig. 1 Representative images of endothelial tube formation assay after treatment with conditioned media from CD34+ (a-c) and CD34-/CD31+ cells (d-f) of endurance-trained ( $\mathbf{a}$ and $\mathbf{d}$ ), active (b and $\mathbf{e}$ ) and inactive (c and $\mathbf{f}$ ) participants. Used with permission [88].

after the intervention, it was again found that exercise training did not affect intracellular redox balance (levels of nitric oxide or reactive oxygen species) in CD34+ CACs.

We then investigated individuals on opposing ends of the health spectrum, i. e., young, healthy, endurance exercise-trained participants vs. older individuals with myocardial infarction [87]. This study was performed to verify previous findings in young healthy populations and to identify potential mechanisms for the differences in S100A8 and S100A9 protein concentrations. As expected, CD34-/CD31 + CM from the healthy group induced longer, more complex endothelial tubes in vitro compared to those of the unhealthy group. This finding was accompanied by a greater concentration of S100A8/A9 in harvested CM from CACs of the unhealthy group. The $\$ 100$ proteins preferentially bind divalent cations such as $\mathrm{Ca}^{2+}$ and have various roles in the regulation of inflammation via reorganization of the actin cytoskeleton, scaffolding and delivery of arachadonic acid, binding to toll-like receptor 4 (TLR4) and receptor for advanced glycation end-products (RAGE), and anti-inflammatory sequestration of IL-1b, IL-6 and TNF $\alpha$ [90]. Because reductions in angiogenesis are accompanied by augmented pro-inflammatory signaling in chronic inflammatory diseases such as CVD, we hypothesized that the $\mathrm{S} 100$ proteins were operating via activation of RAGE or TLR4. This was supported by our in vitro data showing that TLR4, but not RAGE antagonists, rescued impaired angiogenesis due to the presence of S100 proteins. These experiments support a definitive role of other non-progenitor cells in regulating angiogenesis in response to exercise by decreasing the release of pro-inflammatory mediators. Though these experiments implicate a clear role of S100 proteins, the signaling pathways that regulate the release of $\mathrm{S} 100$ s with exercise training are still unclear. Future research investigating the precise cells responsible for the release of S100s in vivo and their molecular cues are warranted for the development of pharmacological interventions and the improvement of cell transplantation efficacy.

\section{CAC-derived microRNA and extracellular vesicles}

Despite the body of literature demonstrating beneficial effects of acute exercise and exercise training on CAC function (see [23] for review), there is a paucity of research on CAC miR expression and release in response to exercise training. The effectiveness of longterm interventions such as lipid lowering therapy in modulating CD34 + /VEGFR2 + cell number and miR content (miR-221 and 222) first suggested a similar potential for aerobic exercise training [91]. Additionally, the body of evidence discussed above shows that various factors/stimuli alter the expression of miRs which regulate paracrine functions of CACs. In one of the first exercise studies, Radom-Aizik et al. [92] reported the effects of 30-minutes of cycling exercise (intervals of 2 minutes of exercise at $\sim 76 \% \mathrm{VO}_{2 \text { peak }}$ and 1 min of rest) on miR expression in the entire peripheral blood mononuclear cell population of young men. This initial analysis showed that 34 miRs were differentially expressed after exercise and 3 miRs (15a, 181a, and 363) were found to be upregulated upon confirmation with RT-qPCR, while miR-451 was downregulated. Given the role of CACs within the overall peripheral blood mononuclear cell population, subsequent studies have begun to focus on miRs and EVs from specific subsets of CACs.

One recent study reported the effects of four weeks of treadmill exercise training ( 15 meters/minute for 60 min, 5 days per week) 
on EPC miRs in four groups of mice [93]. The mice received either a ligation-induced myocardial infarction or sham surgery, then underwent four weeks of exercise training or remaining sedentary. EPC expression of miR-126 was highest in the MI group that received exercise training, and the authors concluded that this likely contributed to enhanced angiogenic function of the EPCs [93]. Another study assessed the effects of four weeks of treadmill exercise on EVs in mice, finding a significantly increased amount of CD34 + $\mathrm{KDR}+$ exosomes in circulation after treadmill exercise training (10 meters/minute for 60 min, 5 days per week) [94]. Furthermore, these exosomes had a significantly higher amount of miR-126 contained within them when compared to the sedentary and low-exercise ( 5 meters/minute for 60 min, 5 days per week) groups. However, there was also a graded effect, as exosomes from the low-exercise group contained higher levels of miR-126 than exosomes from sedentary mice. A concurrent increase was seen in VEGF protein levels in endothelial cells co-cultured with CACs derived from the group that performed moderate-intensity exercise training, implying that this intervention induced protective effects of CACs on endothelial cells. The contribution of miR-126 to these changes was supported when knockdown of miR-126 in exosomes derived from the CACs of moderate intensity exercise trained mice abolished these changes, inducing VEGF levels in co-cultured endothelial cells that mirrored the co-culture group that contained only endothelial cells in co-culture medium (negative control). Similarly, in an animal model of stroke, circulating CD34 + /KDR + exosomes from mice undergoing exercise post-stroke ( 10 meters/minute for $60 \mathrm{~min}, 5$ days per week for 4 weeks) contained greater miR-126 content and had improved microvessel density than their sedentary counterparts [95]. miR-126 content was negatively correlated with stroke outcomes such as infarct volume and cell death. Additionally, the beneficial effects of miR-126 on neuronal outcomes were blocked with PI3k and miR-126 inhibitors. Importantly, in the exercise vs control group, these improvements were augmented at 14 and 28 days of exercise post stroke suggesting that exercise training extended benefits. It should be noted that in these in vivo studies, exercise training likely stimulated a number of pathways involving hypoxia, shear stress and cytokine release to improve angiogenesis and vascular function; however, these studies demonstrate the possibility that aerobic exercise training can beneficially modify the contents of CAC-derived EVs to improve disease outcomes.

In a recent study involving older patients with type 2 diabetes who had been instructed to engage in self-paced physical activity (e. g., walking in one's neighborhood), subjects who had engaged in the highest volume of exercise ( 344 MET-minutes per day) over a three month period showed a significant decrease in miR-146a expression in CACs, while subjects who engaged in a moderate amount of exercise ( 270 MET-minutes per day) experienced a significant increase in miR-130a expression [96]. These changes in intracellular miR levels suggest that exercise training may beneficially modulate CAC paracrine function, as miR-146a is highly upregulated in response to senescence and miR-130a is proangiogenic $[67,76]$. Taken together, these studies suggest the potential of chronic exercise training to beneficially modify miR secretion based on changes in intracellular expression, especially in subjects with existing cardiovascular impairments.
The literature on circulating miRs has been previously reviewed in the context of exercise, training, and fitness level [97]. However, conflicting evidence has been provided on the associations between changes in circulating miR and intracellular CAC miR levels $[73,75,76]$. The levels of expression in these two pools of miRs may be similar, while in other cases may be opposite of one another (i. e., higher in CACs but lower in serum) [75]. In addition, it is difficult to determine which miRs in circulation have been released by CACs, as there is no way to identify the cellular source of circulating miRs. However, CACs do express some miRs that have pro-angiogenic functions and are thought to be endothelial cell-specific [61], supporting the notion that more studies of CAC miR expression and secretion in response to exercise are needed.

Though only one known study to date has been published on the effects of exercise training on CAC EV paracrine release [94], the effects of an acute bout of exercise on CAC-derived EVs have been reported. At various intensities ranging from $\sim 50 \% \mathrm{VO} 2 \mathrm{max}$ to a maximal exercise test, an acute bout of cycle or treadmill exercise is known to increase the release of CAC-derived EVs [98-100]. However, the mechanisms underlying EV release from CACs following an acute bout of exercise are largely unknown, as demonstrated by conflicting findings reported by two similar studies $[99,100]$. While one study reported that the number of EVs derived from CD15 + polymorphonuclear neutrophils, but not from CD14 + monocytes, increased in healthy trained individuals after a bout of maximal exercise, the other found that CD14 + monocytes release a significantly greater amount of EVs following a bout of submaximal exercise $[99,100]$. Taken together, the findings summarized from the above exercise training and acute exercise studies support the potential of chronic exercise to beneficially modulate the paracrine release of miRs, EVs and EV cargo from CACs.

\section{Conclusions}

The paracrine function of CACs is an integral part of their role in angiogenesis and maintenance of the endothelium, whether directly through the secretion of various growth factors or indirectly through inflammatory mediators. While exercise training elicits stimuli such as hypoxia, shear stress and cytokine release that may directly affect angiogenesis and cardiovascular health, the research cited herein indicates exercise training also may elicit these improvements through enhanced CAC paracrine function ( $\triangleright \mathbf{F i g . ~ 2}$ ). Though nascent, the research in this area has already identified roles of the S100A proteins and miRs- 126,130 , and 146a as effectors of the benefits of exercise on CAC paracrine function. Still, many factors identified as important in CAC paracrine function have not yet been investigated in regard to exercise training.

First and foremost, there is a general lack of longitudinal studies assessing CAC paracrine function following exercise training; future prospective trials are essential to improve our understanding of the effects of exercise training in a variety of populations. Second, these studies should strive to define the precise molecular, mechanical or inflammatory cues that elicit beneficial changes with exercise training. Given the wide range of potential responses and the fact that multiple cells and tissues are affected by exercise training, it is also likely that mechanisms of cellular cross-talk, such as CAC-EVs, are involved and warrant investigation. Third, future 

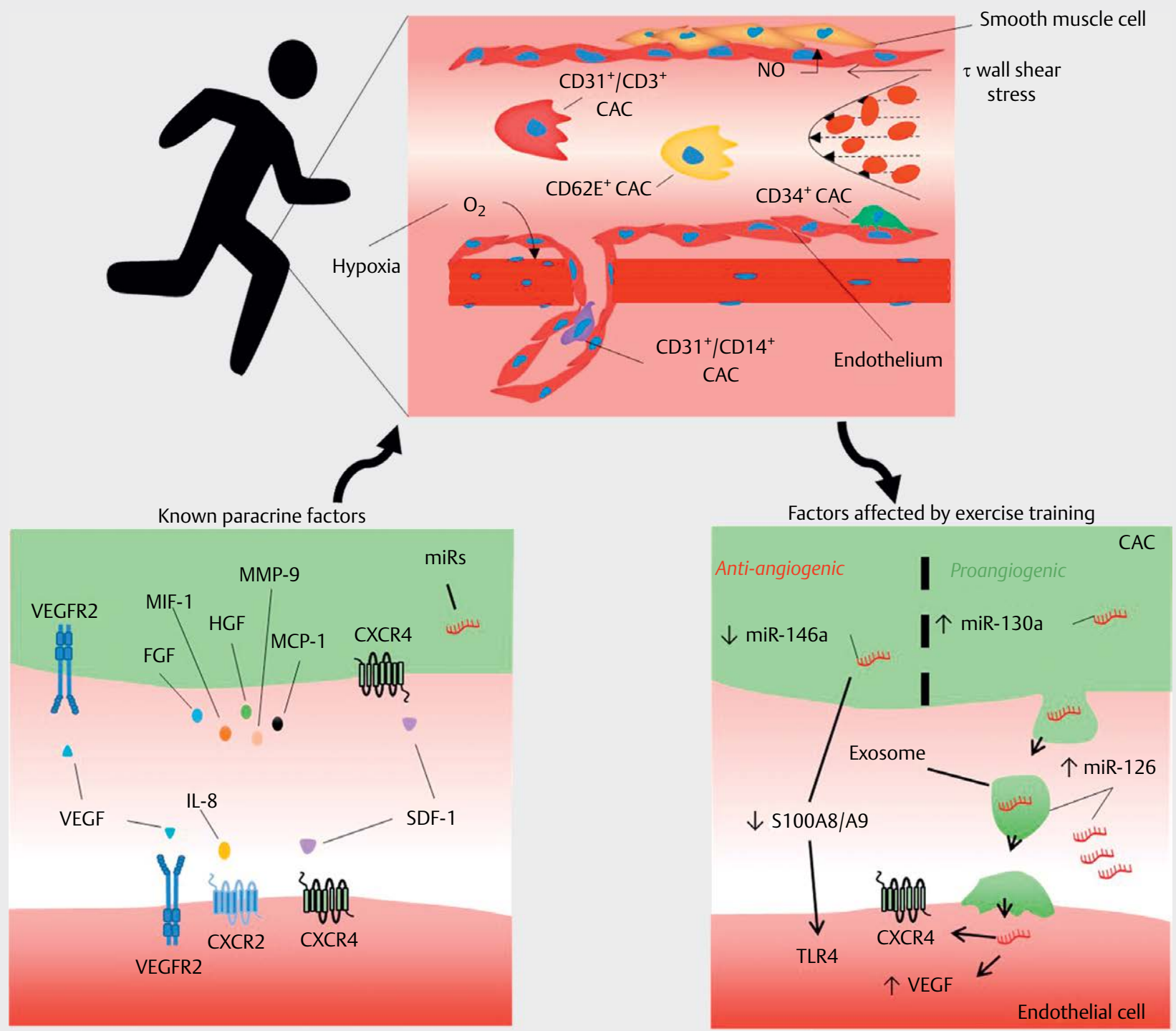

Fig. 2 Conceptual figure of changes in CAC paracrine function as a result of exercise training. Circulating angiogenic cells (CAC) adhere to the endothelium (top panel) and secrete paracrine factors to promote angiogenesis. Exercise training (bottom right panel) increases CAC release of miR-126, both within and independent of exosomes/extracellular vesicles (EV), which enters endothelial cells and increases expression of VEGF and CXCR4 by targeting inhibitors of their production. Exercise training also decreases CAC expression of miR-146a (anti-angiogenic) and increases miR130-a (pro-angiogenic) while promoting a decrease in S100A8/A9 ratio (ameliorating an inflammatory response). Stimuli associated with exercise training such as NO release, wall shear stress, and hypoxia are known to affect CAC paracrine function (top panel), but these changes have not been directly linked to CAC secretion of various growth factors, cytokines, chemokines and miRs with exercise training (bottom left panel).

research should examine specific subsets of CACs to better understand the unique functions of each subset and the influence of exercise training on paracrine function. We currently understand that exercise training affects, and often increases, the number of cells within certain subpopulations of CACs (for review see [23]), the response is not uniform across all subpopulations and this differential response may result in distinct CAC subpopulations having different contributions to overall CAC paracrine function.

Finally, the aforementioned factors and mechanisms should also be explored in the context of common cardiometabolic diseases known to affect CACs and angiogenesis, such as coronary heart disease and type 2 diabetes mellitus. Studies of autologous circulating stem/progenitor cell therapies have been underway in these populations for the past 2 decades, but results are often inconclusive. In our view, exercise training studies could be particularly useful in these groups to identify optimal CAC subpopulations and paracrine mechanisms that could be targeted for enhancement prior to autologous cell therapies. Furthermore, the effects of different types and intensities of exercise, as well as the independent and combined effects of exercise and weight loss, require study in an attempt to de- 
fine optimal exercise prescriptions to enhance CAC and vascular function, with the effects of strength vs. aerobic exercise or continuous vs. high intensity interval training being ripe for study. While current exercise guidelines focus mainly on achieving a number of minutes of moderate or vigorous aerobic exercise each week, identifying optimal frequencies, intensities, durations and types of aerobic or strength exercise to enhance CAC paracrine function could lead to better personalization of exercise prescription to preserve or enhance cardiovascular function in health and disease.

In summary, the research reviewed herein establishes a role of CAC paracrine function in promoting angiogenesis and identifies several proteins and miRs that appear to mediate the beneficial effects of exercise training on CAC paracrine function. These early studies provide promising results and lay the framework and rationale for future studies to fully elucidate the effects of exercise training on CAC paracrine function.

\section{Acknowledgements}

Dr. Prior was supported by the Department of Veterans Affairs Baltimore Geriatric Research, Education and Clinical Center.

\section{Conflict of Interest}

The authors have no conflict of interest to disclose. This work meets the ethical standards established by the International Journal of Sports Medicine [101].

\section{References}

[1] Sietsema WK, Kawamoto A, Takagi H et al. Autologous CD34 + cell therapy for ischemic tissue repair. Circ J 2019; 83: 1422-1430

[2] Kinnaird T, Stabile E, Burnett MS et al. Marrow-derived stromal cells express genes encoding a broad spectrum of arteriogenic cytokines and promote in vitro and in vivo arteriogenesis through paracrine mechanisms. Circ Res 2004; 94: 678-685

[3] Di Santo S, Yang Z, Wyler von Ballmoos M et al. Novel cell-free strategy for therapeutic angiogenesis: In vitro generated conditioned medium can replace progenitor cell transplantation. PLoS One 2009; 4: e5643

[4] Zafar N, Krishnasamy SS, Shah J et al. Circulating angiogenic stem cells in type 2 diabetes are associated with glycemic control and endothelial dysfunction. PLoS One 2018; 13: e0205851

[5] Vasa M, Fichtlscherer S, Aicher A et al. Number and migratory activity of circulating endothelial progenitor cells inversely correlate with risk factors for coronary artery disease. Circ Res 2001; 89: E1-E7

[6] Cavalcante SL, Lopes S, Bohn L et al. Effects of exercise on endothelial progenitor cells in patients with cardiovascular disease: A systematic review and meta-analysis of randomized controlled trials. Rev Port Cardiol 2019; 38: 817-827

[7] Rehman J, Li J, Orschell CM et al. Peripheral blood "endothelial progenitor cells" are derived from monocyte/macrophages and secrete angiogenic growth factors. Circulation 2003; 107: 1164-1169

[8] Rohde E, Malischnik C, Thaler D et al. Blood monocytes mimic endothelial progenitor cells. Stem Cells 2006; 24: 357-367

[9] Hur ], Yang H-M, Yoon C-H et al. Identification of a novel role of T cells in postnatal vasculogenesis. Circulation 2007; 116: 1671-1682
[10] Iwase T, Nagaya N, Fujii T et al. Comparison of angiogenic potency between mesenchymal stem cells and mononuclear cells in a rat model of hindlimb ischemia. Cardiovasc Res 2005; 66: 543-551

[11] Fadini GP, Losordo D, Dimmeler S. Critical reevaluation of endothelial progenitor cell phenotypes for therapeutic and diagnostic use. Circ Res 2012; 110: 624-637

[12] Hill JM, Zalos G, Halcox JP et al. Circulating endothelial progenitor cells, vascular function, and cardiovascular risk. N Engl J Med 2003; 348: $593-600$

[13] Asahara T, Murohara T, Sullivan A et al. Isolation of putative progenitor endothelial cells for angiogenesis. Science 1997; 275: 964-967

[14] Bielak LF, Horenstein RB, Ryan KA et al. Circulating CD34 + cell count is associated with extent of subclinical atherosclerosis in asymptomatic amish men, independent of 10-year framingham risk. Clin Med Cardiol 2009; 3: 53-60

[15] Kim S-W, Kim H, Cho H-J et al. Human peripheral blood-derived CD31 + cells have robust angiogenic and vasculogenic properties and are effective for treating ischemic vascular disease. J Am Coll Cardiol 2010; 56: 593-607

[16] Kim S-W, Kim H, Yoon Y-s. Advances in bone marrow-derived cell therapy: CD31-expressing cells as next generation cardiovascular cell therapy. Regen Med 2011; 6: 335-349

[17] Erdbruegger U, Dhaygude A, Haubitz M et al. Circulating endothelial cells: markers and mediators of vascular damage. Curr Stem Cell Res Ther 2010; 5: 294-302

[18] Goon PKY, Boos C], Stonelake PS et al. Detection and quantification of mature circulating endothelial cells using flow cytometry and immunomagnetic beads: A methodological comparison. Thromb Haemost 2006; 96: 45-52

[19] Kushner EJ, MacEneaney OJ, Morgan RG et al. CD31 + T cells represent a functionally distinct vascular $\mathrm{T}$ cell phenotype. Blood Cells Mol Dis 2010; 44: 74-78

[20] Hur ], Yang HM, Yoon CH et al. Identification of a novel role of T cells in postnatal vasculogenesis: characterization of endothelial progenitor cell colonies. Circulation 2007; 116: 1671-1682

[21] Kim S-J, Kim J-S, Papadopoulos ] et al. Circulating monocytes expressing CD31: Implications for acute and chronic angiogenesis. Am J Pathol 2009; 174: 1972-1980

[22] Waller E, Olweus J, Lund-Johansen F et al. The "common stem cell" hypothesis reevaluated: human fetal bone marrow contains separate populations of hematopoietic and stromal progenitors. Blood 1995; 85: 2422-2435

[23] Landers-Ramos RQ, Sapp RM, Shill DD et al. Exercise and cardiovascular progenitor cells. Compr Physiol 2019; 9: 767-797

[24] Khan SS, Solomon MA, McCoy JP Jr. Detection of circulating endothelial cells and endothelial progenitor cells by flow cytometry. Cytometry B Clin Cytom 2006; 70: 104-105

[25] Lertkiatmongkol P, Liao D, Mei $\mathrm{H}$ et al. Endothelial functions of platelet/endothelial cell adhesion molecule-1 (CD31). Curr Opin Hematol 2016; 23: 253-259

[26] Ziegelhoeffer T, Fernandez B, Kostin S et al. Bone marrow-derived cells do not incorporate into the adult growing vasculature. Circ Res 2004; 94: 230-238

[27] Gnecchi M, Zhang Z, Ni A et al. Paracrine mechanisms in adult stem cell signaling and therapy. Circ Res 2008; 103: 1204-1219

[28] Yang Z, von Ballmoos MW, Faessler D et al. Paracrine factors secreted by endothelial progenitor cells prevent oxidative stress-induced apoptosis of mature endothelial cells. Atherosclerosis 2010; 211: 103-109

[29] Xu S, Zhu J, Yu L et al. Endothelial progenitor cells: Current development of their paracrine factors in cardiovascular therapy. J Cardiovasc Pharmacol 2012; 59: 387-396 
[30] Bollini S, Gentili C, Tasso R et al. The Regenerative role of the fetal and adult stem cell secretome. J Clin Med 2013; 2: 302-327

[31] Kamihata $\mathrm{H}$, Matsubara $\mathrm{H}$, Nishiue $\mathrm{T}$ et al. Implantation of bone marrow mononuclear cells into ischemic myocardium enhances collateral perfusion and regional function via side supply of angioblasts, angiogenic ligands, and cytokines. Circulation 2001; 104: 1046-1052

[32] Urbich C, Aicher A, Heeschen C et al. Soluble factors released by endothelial progenitor cells promote migration of endothelial cells and cardiac resident progenitor cells. J Mol Cell Cardiol 2005; 39: 733-742

[33] Asahara T, Masuda H, Takahashi T et al. Bone marrow origin of endothelial progenitor cells responsible for postnatal vasculogenesis in physiological and pathological neovascularization. Circ Res 1999; 85: 221-228

[34] Akita $\mathrm{T}$, Murohara $\mathrm{T}$, Ikeda $\mathrm{H}$ et al. Hypoxic preconditioning augments efficacy of human endothelial progenitor cells for therapeutic neovascularization. Lab Invest 2003; 83: 65-73

[35] Medina RJ, O'Neill CL, O'Doherty TM et al. Myeloid angiogenic cells act as alternative $\mathrm{M} 2$ macrophages and modulate angiogenesis through interleukin-8. Mol Med 2011; 17: 1045-1055

[36] Medina RJ, O’Neill CL, Sweeney M et al. Molecular analysis of endothelial progenitor cell (EPC) subtypes reveals two distinct cell populations with different identities. BMC Med Genomics 2010; 3: 18

[37] Zemani F, Silvestre JS, Fauvel-Lafeve F et al. Ex vivo priming of endothelial progenitor cells with SDF-1 before transplantation could increase their proangiogenic potential. Arterioscler Thromb Vasc Biol 2008; 28: 644-650

[38] Odent Grigorescu G, Rosca AM, Preda MB et al. Synergic effects of VEGF-A and SDF-1 on the angiogenic properties of endothelial progenitor cells. J Tissue Eng Regen Med 2017; 11: 3241-3252

[39] Yin Y, Huang L, Zhao X et al. AMD3100 mobilizes endothelial progenitor cells in mice, but inhibits its biological functions by blocking an autocrine/paracrine regulatory loop of stromal cell derived factor-1 in vitro. J Cardiovasc Pharmacol 2007; 50: 61-67

[40] Grunewald M, Avraham I, Dor Y et al. VEGF-induced adult neovascularization: recruitment, retention, and role of accessory cells. Cell 2006; 124: 175-189

[41] Pula G, Mayr U, Evans C et al. Proteomics identifies thymidine phosphorylase as a key regulator of the angiogenic potential of colony-forming units and endothelial progenitor cell cultures. Circ Res 2009; 104: 32-40

[42] Urbich C, De Souza Al, Rossig L et al. Proteomic characterization of human early pro-angiogenic cells. J Mol Cell Cardiol 2011; 50: 333-336

[43] Felice F, Piras AM, Rocchiccioli S et al. Endothelial progenitor cell secretome delivered by novel polymeric nanoparticles in ischemic hindlimb. Int J Pharm 2018; 542: 82-89

[44] Wara AK, Foo S, Croce $\mathrm{K}$ et al. TGF-beta1 signaling and Kruppel-like factor 10 regulate bone marrow-derived proangiogenic cell differentiation, function, and neovascularization. Blood 2011; 118: 6450-6460

[45] Tan Q, Zhang S, Qi X et al. Permanent atrial fibrillation impairs the function of circulating endothelial progenitor cells. Postgrad Med 2017; 129: 198-204

[46] Teraa M, Sprengers RW, Westerweel PE et al. Bone marrow alterations and lower endothelial progenitor cell numbers in critical limb ischemia patients. PLoS One 2013; 8: e55592

[47] Heida N-M, Müller J-P, Cheng IF et al. Effects of obesity and weight loss on the functional properties of early outgrowth endothelial progenitor cells. J Am Coll Cardiol 2010; 55: 357-367

[48] Jarajapu YPR, Hazra S, Segal M et al. Vasoreparative dysfunction of CD34 + cells in diabetic individuals involves hypoxic desensitization and impaired autocrine/paracrine mechanisms. PLoS One 2014; 9: e93965
[49] Kanzler I, Tuchscheerer N, Steffens G et al. Differential roles of angiogenic chemokines in endothelial progenitor cell-induced angiogenesis. Basic Res Cardiol 2013; 108: 310

[50] Wu Y, Li L, Chen W et al. Maintaining Moderate platelet aggregation and improving metabolism of endothelial progenitor cells increase the patency rate of tissue-engineered blood vessels. Tissue Eng Part A 2015; 21: 2001-2012

[51] Han J-K, Kim H-L, Jeon K-H et al. Peroxisome proliferator-activated receptor- $\delta$ activates endothelial progenitor cells to induce angiomyogenesis through matrix metallo-proteinase-9-mediated insulin-like growth factor-1 paracrine networks. Eur Heart ] 2011; 34: 1755-1765

[52] Leifheit-Nestler M, Conrad G, Heida NM et al. Overexpression of integrin beta 5 enhances the paracrine properties of circulating angiogenic cells via Src kinase-mediated activation of STAT3. Arterioscler Thromb Vasc Biol 2010; 30: 1398-1406

[53] Wara AK, Manica A, Marchini JF et al. Bone marrow-derived Kruppel-like factor 10 controls reendothelialization in response to arterial injury. Arterioscler Thromb Vasc Biol 2013; 33: 1552-1560

[54] Scheubel RJ, Holtz J, Friedrich I et al. Paracrine effects of CD34 progenitor cells on angiogenic endothelial sprouting. Int J Cardiol 2010; 139: 134-141

[55] Burlacu A, Grigorescu G, Rosca AM et al. Factors secreted by mesenchymal stem cells and endothelial progenitor cells have complementary effects on angiogenesis in vitro. Stem Cells Dev 2013; 22: 643-653

[56] Krenning G, van der Strate BWA, Schipper M et al. Combined implantation of CD34 + and CD14 + cells increases neovascularization through amplified paracrine signalling. J Tissue Eng Regen Med 2013; 7: 118-128

[57] Jackson RJ, Standart $\mathrm{N}$ how do micrornas regulate gene expression? SCi STKE 2007; 2007: re1

[58] Carthew RW, Sontheimer EJ. Origins and mechanisms of miRNAs and siRNAs. Cell 2009; 136: 642-655

[59] Fabian MR, Sonenberg N. The mechanics of miRNA-mediated gene silencing: a look under the hood of miRISC. Nat Struct Mol Biol 2012; 19: 586-593

[60] Chistiakov DA, Orekhov AN, Bobryshev YV. The role of miR-126 in embryonic angiogenesis, adult vascular homeostasis, and vascular repair and its alterations in atherosclerotic disease. J Mol Cell Cardiol 2016; 97: 47-55

[61] Fish JE, Santoro MM, Morton SU et al. miR-126 regulates angiogenic signaling and vascular integrity. Dev Cell 2008; 15: 272-284

[62] Shan C, Ma Y. MicroRNA-126/stromal cell-derived factor 1/C-X-C chemokine receptor type 7 signaling pathway promotes post-stroke angiogenesis of endothelial progenitor cell transplantation. Mol Med Rep 2018; 17: 5300-5305

[63] Zhang J, Zhang Z, Zhang DY et al. microRNA 126 inhibits the transition of endothelial progenitor cells to mesenchymal cells via the PIK3R2-PI3K/Akt signalling pathway. PLoS One 2013; 8: e83294

[64] Pan Q, Zheng J, Du D et al. MicroRNA-126 priming enhances functions of endothelial progenitor cells under physiological and hypoxic conditions and their therapeutic efficacy in cerebral ischemic damage. Stem Cells Int 2018; 2018: 2912347

[65] Li Y, Zhou Q, Pei C et al. Hyperglycemia and advanced glycation end products regulate miR-126 expression in endothelial progenitor cells. J Vascular Res 2016; 53: 94-104

[66] Jakob P, Doerries C, Briand S et al. Loss of AngiomiR-126 and 130a in angiogenic early outgrowth cells from patients with chronic heart failure. Circulation 2012; 126: 2962-2975

[67] Meng S, Cao J, Zhang X et al. Downregulation of microRNA-130a contributes to endothelial progenitor cell dysfunction in diabetic patients via its target Runx3. PLoS One 2013; 8: e68611 
[68] Weber M, Baker MB, Moore JP et al. MiR-21 is induced in endothelial cells by shear stress and modulates apoptosis and eNOS activity. Biochem Biophys Res Commun 2010; 393: 643-648

[69] Zuo K, Li M, Zhang X et al. MiR-21 suppresses endothelial progenitor cell proliferation by activating the TGF $\beta$ signaling pathway via downregulation of WWP1. Int J Clin Exp Pathol 2015; 8: 414-422

[70] Fleissner F, Jazbutyte V, Fiedler J et al. Short communication: asymmetric dimethylarginine impairs angiogenic progenitor cell function in patients with coronary artery disease through a microRNA-21-dependent mechanism. Circ Res 2010; 107: 138-143

[71] Thum T, Tsikas D, Stein S et al. Suppression of endothelial progenitor cells in human coronary artery disease by the endogenous nitric oxide synthase inhibitor asymmetric dimethylarginine. J Am Coll Cardiol 2005; 46: 1693-1701

[72] Zhang HW, Li H, Yan H et al. MicroRNA-142 promotes the expression of eNOS in human peripheral blood-derived endothelial progenitor cells in vitro. Eur Rev Med Pharmacol Sci 2016; 20: 4167-4175

[73] Spinetti G, Fortunato O, Caporali A et al. MicroRNA-15a and MicroRNA-16 impair human circulating proangiogenic cell functions and are increased in the proangiogenic cells and serum of patients with critical limb ischemia. Circ Res 2013; 112: 335-346

[74] Goretti E, Rolland-Turner M, Leonard F et al. MicroRNA-16 affects key functions of human endothelial progenitor cells. J Leukoc Biol 2013; 93: 645-655

[75] Wang H-W, Lo H-H, Chiu Y-L et al. Dysregulated miR-361-5p/VEGF axis in the plasma and endothelial progenitor cells of patients with coronary artery disease. PLoS One 2014; 9: e98070

[76] Olivieri F, Lazzarini R, Recchioni R et al. MiR-146a as marker of senescence-associated pro-inflammatory status in cells involved in vascular remodelling. Age (Dordr) 2013; 35: 1157-1172

[77] Thery C, Ostrowski M, Segura E. Membrane vesicles as conveyors of immune responses. Nat Rev Immunol 2009; 9: 581-593

[78] Gould S], Raposo G. As we wait: coping with an imperfect nomenclature for extracellular vesicles. J Extracell Vesicles 2013; 2: 20389

[79] van Niel G, D’Angelo G, Raposo G. Shedding light on the cell biology of extracellular vesicles. Nat Rev Mol Cell Biol 2018; 19: 213-228

[80] Deregibus MC, Cantaluppi V, Calogero R et al. Endothelial progenitor cell-derived microvesicles activate an angiogenic program in endothelial cells by a horizontal transfer of mRNA. Blood 2007; 110: 2440-2448

[81] Mathiyalagan P, Liang Y, Kim D et al. Angiogenic mechanisms of human CD34(+) stem cell exosomes in the repair of ischemic hindlimb. Circ Res 2017; 120: 1466-1476

[82] Sahoo S, Klychko E, Thorne T et al. Exosomes from human CD34(+) stem cells mediate their proangiogenic paracrine activity. Circ Res 2011; 109: 724-728

[83] Hristov M, Erl W, Linder S et al. Apoptotic bodies from endothelial cells enhance the number and initiate the differentiation of human endothelial progenitor cells in vitro. Blood 2004; 104: 2761-2766

[84] Zernecke A, Bidzhekov K, Noels $\mathrm{H}$ et al. Delivery of microRNA- 126 by apoptotic bodies induces CXCL12-dependent vascular protection. Sci Signal 2009; 2: ra81

[85] Wu K, Yang $Y$, Zhong $Y$ et al. The effects of microvesicles on endothelial progenitor cells are compromised in type 2 diabetic patients via downregulation of the miR-126/VEGFR2 pathway. Am J Physiol Endocrinol Metab 2016; 310: E828-E837
[86] Yue Y, Garikipati VNS, Verma SK et al. Interleukin-10 deficiency impairs reparative properties of bone marrow-derived endothelial progenitor cell exosomes. Tissue Eng Part A 2017; 23: 1241-1250

[87] Landers-Ramos RQ, Sapp RM, VandeWater E et al. Investigating the extremes of the continuum of paracrine functions in CD34-/CD31+ CACs across diverse populations. Am J Physiol Heart Circ Physiol 2017; 312: H162-H172

[88] Landers-Ramos RQ, Sapp RM, Jenkins NT et al. Chronic endurance exercise affects paracrine action of CD31 + and CD34 + cells on endothelial tube formation. Am J Physiol Heart Circ Physiol 2015; 309: $\mathrm{H} 407-\mathrm{H} 420$

[89] Landers-Ramos RQ, Corrigan KJ, Guth LM et al. Short-term exercise training improves flow-mediated dilation and circulating angiogenic cell number in older sedentary adults. Appl Physiol Nutr Metab 2016; 41: 832-841

[90] Wang S, Song R, Wang Z et al. S100A8/A9 in Inflammation. Front Immunol 2018; 9: 1298

[91] Minami Y, Satoh M, Maesawa C et al. Effect of atorvastatin on microRNA 221/222 expression in endothelial progenitor cells obtained from patients with coronary artery disease. Eur ] Clin Invest 2009; 39: 359-367

[92] Radom-Aizik S, Zaldivar F Jr., Leu SY et al. Effects of exercise on microRNA expression in young males peripheral blood mononuclear cells. Clin Transl Sci 2012; 5: 32-38

[93] Guo Y, Luo F, Zhang X et al. TPPU enhanced exercise-induced epoxyeicosatrienoic acid concentrations to exert cardioprotection in mice after myocardial infarction. J Cell Mol Med 2018; 22: 14891500

[94] Ma C, Wang J, Liu H et al. Moderate exercise enhances endothelial progenitor cell exosomes release and function. Med Sci Sports Exerc 2018; 50: 2024-2032

[95] Wang J, Liu H, Chen $\mathrm{S}$ et al. Moderate exercise has beneficial effects on mouse ischemic stroke by enhancing the functions of circulating endothelial progenitor cell-derived exosomes. Exp Neurol 2020; 330: 113325

[96] Cirilli I, Silvestri S, Marcheggiani F et al. Three Months monitored metabolic fitness modulates cardiovascular risk factors in diabetic patients. Diabetes Metab J 2019; 43: 893-897

[97] Sapp RM, Shill DD, Roth SM et al. Circulating microRNAs in acute and chronic exercise: more than mere biomarkers. J Appl Physiol (1985) 2016: 702-717

[98] Lansford KA, Shill DD, Dicks AB et al. Effect of acute exercise on circulating angiogenic cell and microparticle populations. Exp Physiol 2016; 101: 155-167

[99] Chaar V, Romana M, Tripette J et al. Effect of strenuous physical exercise on circulating cell-derived microparticles. Clin Hemorheol Microcirc 2011; 47: 15-25

[100] Sossdorf M, Otto GP, Claus RA et al. Cell-derived microparticles promote coagulation after moderate exercise. Med Sci Sports Exerc 2011; 43: 1169-1176

[101] Harriss D], MacSween A, Atkinson G. Ethical standards in sport and exercise science research: 2020 update. Int ] Sports Med 2019; 40: 813-817

[102] Zhu S, Deng S, Ma Q et al. MicroRNA-10A* and MicroRNA-21 modulate endothelial progenitor cell senescence via suppressing high-mobility group A2. Circ Res 2013; 112: 152-164 